\title{
Association of the polymorphisms of MMP-9 and TIMP-3 genes with thoracic aortic dissection in Chinese Han population
}

\author{
Xiao-long WANG ${ }^{1,2, \#}$, Ou LIU 2, 3, \#, Yan-wen QIN², Hong-jia ZHANG', *, Yi LV1, 2, * \\ ${ }^{1}$ Department of Hepatobiliary Surgery, the First Affiliated Hospital of Medical College, Xi-an Jiaotong University, Xi-an 710000, China; \\ ${ }^{2}$ Department of Cardiovascular Surgery, Beijing An Zhen Hospital, Capital Medical University, Beijing 100029, China; ${ }^{3}$ The Key Labora- \\ tory of Remodeling-related Cardiovascular Diseases, Capital Medical University, Ministry of Education, Beijing 100029, China
}

Aim: Thoracic aortic dissection (TAD) is the most common life-threatening disorder, and a shifted balance of matrix metalloproteinases (MMPs) and tissue inhibitors of metalloproteinases (TIMPS) is involved in TAD pathogenesis. The aim of this study was to evaluate the association of 4 single-nucleotide polymorphisms (SNPS) in MMP-9 and TIMP-3 genes with TAD risk in Chinese Han population. Methods: A total of 206 Chinese patients with TAD and 180 controls were included in this study. Four SNPs (rs3918249, rs2274756, rs9609643 and rs8136803) were genotyped using high-throughput MALDI-TOF mass spectrometry. Allele and genotype association analyses were conducted using PLINK.

Results: All the 4 SNPs resulted in Hardy-Weinberg equilibrium in patients and controls. The G allele frequency for the MMP-9 SNP rs2274756 was significantly higher in female TAD patients than in female controls $(P=0.0099)$. Moreover, after adjusting for traditional cardiovascular risk factors (sex, age, hypertension, dyslipidemia, diabetes and smoking habit), the rs2274756 polymorphism (odds ratio: 0.30 ; $95 \%$ confidence interval: 0.11 to $0.79, P=0.015)$ resulted in an independent susceptibility factor for TAD in females. No associations were found between the other SNPs and TAD.

Conclusion: The results provide strong evidence for an association between MMP-9 SNP rs2274756 and female TAD risk in Chinese Han population.

Keywords: aortic dissection; genetic variation; SNP; MMP-9; TIMP-3; Chinese population

Acta Pharmacologica Sinica (2014) 35: 351-355; doi: 10.1038/aps.2013.179; published online 3 Feb 2014

\section{Introduction}

Thoracic aortic dissection (TAD) is the most common lifethreatening disorder that affects the aorta in the world ${ }^{[1]}$. Evidence from clinical trials indicates that the mortality rate from acute aortic dissection is as high as $1 \%$ per hour in the early stages ${ }^{[2]}$, making prevention and early diagnosis critical for survival. Studies on the pathogenesis of this disease may shed light on the prevention and treatment of dissection. It is believed that hypertension is the main etiological risk factor in aortic dissection development ${ }^{[3]}$. Meanwhile, a number of genetic conditions are also associated with dissection, such as Marfan syndrome_ENREF_1 $1^{[4]}$ and Loeys-Dietz syndrome ${ }^{[5]}$. However, the cause of spontaneous aortic dissection in indi-

\footnotetext{
\# These authors contributed equally to this work.

* To whom correspondence should be addressed.

E-mail zhanghongjia722@hotmail.com (Hong-jia ZHANG); luyi169@126.com (Yi LV)

Received 2013-07-06 Accepted 2013-11-06
}

viduals without genetic syndromes is still unknown.

The histological appearance of aortic dissection is characterized by aortic wall inflammation and the progressive degradation of extracellular matrix proteins due to an imbalance of proteolytic enzymes and their inhibitors ${ }^{[6]}$. A number of proteolytic enzymes are involved in the development of aortic dissection, namely, matrix metalloproteinases (MMPs) and tissue inhibitors of metalloproteinases (TIMPs) ${ }^{[7]}$. MMPs comprise a family of over 20 zinc-dependent enzymes that degrade all major protein components of the extracellular matrix $(\mathrm{ECM})^{[8]}$. TIMPs are endogenous inhibitors of activated MMPs that keep their activity in check ${ }^{[9]}$. The interaction between MMPs and TIMPs has been reported to play a crucial role in pathogenesis of aortic dissection and other tissue remodeling-related diseases, such as primary angle closure glaucoma (PACG) ${ }^{[10]}$ and tumor ${ }^{[11]}$. Recently, four MMP-9 and TIMP-3 singlenucleotide polymorphisms (SNPs) have been identified to be associated with the pathogenesis of $\operatorname{PACG}^{[12]}$ and cancer ${ }^{[13,14]}$ in several studies. SNPs are the most common genetic varia- 
tion in the human genome, which could explain differences in genetic susceptibility to complex disease ${ }^{[15]}$. It remains unclear whether these four SNPs that are associated with some tissue remodeling-related diseases could influence the formation of TAD.

In the current study, we aimed to assess the four SNPs in the MMP-9 and TIMP-3 genes for their association with TAD in the Chinese Han population.

\section{Materials and methods Study population}

We carried out a case-control association study involving 206 unrelated cases that were randomly selected from patients admitted to Beijing Anzhen Hospital with a diagnosis of TAD. The diagnostic criteria for TAD were as described previously $^{[16]}$. In brief, diagnosis was based on any type of imaging examination, including CT scan, MRI, echocardiography, and angiography. Patients with Marfan syndrome, Loeys-Dietz aneurysm, aortic coarctation, or other aortic diseases were excluded from the study.

The control group consisted of 180 volunteers randomly selected from individuals who were admitted to Beijing Anzhen Hospital for reasons other than aortic disease, mainly primary hypertension disease. The aorta was routinely measured by echocardiography for hypertensive patients in our hospital. Control patients were enrolled only if angiography revealed no evidence of aortic diseases, such as aortic dissection and abnormal aortic diameter. We chose hypertensive for the control group because it is known that hypertension is an important risk factor for TAD, and this control group eliminated the potential contribution of hypertension to TAD. Case and control samples were matched by ethnic ancestry (all Chinese Han population) and age. Information on demographic characteristics was collected, and clinical information and family histories were collected from medical records and interviews that were conducted by a genetics counselor. All protocols involving human specimens were approved by the Institutional Review Board at Beijing Anzhen Hospital. Each subject provided written informed consent.

\section{Blood sample collection and genomic DNA extraction}

Ethylenediamine tetraacetic acid (EDTA) anticoagulated venous whole blood samples were collected from each participant. Human genomic DNA was isolated from peripheral blood leukocytes by standard procedures using the DNA Isolation Kit (Genomic DNA kit, Axygen Scientific Inc, CA, USA), and the DNA was stored at $-80^{\circ} \mathrm{C}$ for further SNP analysis.

\section{Genotyping}

We studied four SNPs of the MMP-9 and TIMP-3 genes associated with either primary angle closure glaucoma and/or cancer based on literature data. This information on SNPS was acquired from the ENSEMBL, dbSNP NCBI and UCSC genome browser databases (Table 1). Of note, reference SNP id rs2274756 was recently merged to rs17577 dbSNP, but for better comparability with previous publications, we continue to refer to this SNP as rs2274756 in the text.

Genotyping analysis of the SNPs was performed using the MassARRAY platform (Sequenom, San Diego, CA, USA). Briefly, polymerase chain reaction (PCR) and detection primers were designed using the MassARRAY Assay Design software (Sequenom). The DNA samples were amplified by multiplex PCR reactions. The terminator nucleotides were desalted and added into a 384-element SpectroCHIP array. Then, allele detection was performed using a time-of-flight (MALDI-TOF) SpectroReader mass spectrometer (Sequenom). Lastly, the mass spectrograms were analyzed for peak identification using the Typer Analyzer software (Sequenom), and for quality control, the missing genotype rate of each SNP was set to lower than $10 \%$.

\section{Statistical analysis}

All analyses were performed using the PLINK 1.07 software. SNPs were tested for deviation from Hardy-Weinberg equilibrium (HWE) using chi-square tests. Continuous data were compared with the use of 2-sample $t$-tests, and categorical data were compared between groups using chi-square tests. Genetic association was assessed under allelic and different genetic models with chi-Square tests, Cochran-Armitage tests for trends and Fisher's exact tests. Where more than 5 counts for a given genotype were observed, a chi-square test was used; otherwise, Fisher's exact test was used. The Bonferroni correction was also applied, and we used logistic regression analysis to test the independence of association evidence between SNPs and TAD, adjusting for traditional cardiovascular risk factors (age, sex, smoking habit, diabetes, hypertension, and dyslipidemia).

\section{Results \\ Study participants}

Table 2 shows the baseline and clinical characteristics of the subjects. We found a statistically significant gender differ-

Table 1. Description of and information for the four investigated single-nucleotide polymorphisms (SNPs). Chr, chromosome.

\begin{tabular}{lccc}
\hline $\begin{array}{l}\text { Gene and ensemble } \\
\text { gene number }\end{array}$ & dbSNP ID & Chr position & Region \\
\hline MMP-9 & rs3918249 & Chromosome 20:44638136 & Intron 1 \\
ENSG00000100985 & rs2274756 & Chromosome 20:44643111 & Exon 12 \\
TIMP-3 & rs9609643 & Chromosome 22:33251059 & G/A \\
ENSG00000100234 & rs8136803 & Chromosome 22:33237112 2 & G/A \\
\hline
\end{tabular}


ence in the occurrence of TAD that was similar to previous reports $^{[17]}$. There were significantly more male than female TAD patients were included in this study. No differences in cardiovascular risk factors such as smoking habit, hypertension, and dyslipidemia between TAD cases and the controls were observed.

\section{Total sample SNP analysis}

All SNPs did not significantly $(P>0.05)$ deviate from HardyWeinberg equilibrium. In Table 3 , the allele frequencies and genotype distributions of all SNPs and the chi-square analysis are reported. We found no significant association between the four SNPs and the TAD phenotype. After adjusting for age, gender, hypertension, diabetes, dyslipidemia, and smoking habit, we performed multiple logistic regression analysis, but no evidence for an association was observed (data not shown).

\section{Gender-stratified sample SNP analysis}

We stratified the samples according to gender to detect possible interactions between SNP genotype status and gender. We did not find significant differences in genotype or allele frequencies between cases and controls in subsamples of males or females for SNP rs3918249, rs9609643, or rs8136803. However, we found a SNP of MMP-9, tagged by rs2274756, in a higher proportion of $\mathrm{G}$ allele carriers among female TAD patients $(93.7 \%)$ compared with female controls $(82.6 \%, P=0.00988)$. Then, we performed a Bonferroni test to calculate the corrected $P$ value. Again, the corrected $P$ value was significant $(P=0.03951)$. Furthermore, genotype distribution resulted in
Table 2. Demographic and clinical characteristics of TAD cases and healthy controls in a Han Chinese population. TAD, thoracic aortic dissection; CAD, coronary artery disease; NS, not significant. Continuous data were tested using a 2-tailed Student's $t$ test, and categorical data were tested using a chi-square test (with $d f=1$ ) for differences between the TAD (patient) and control (normal) groups.

\begin{tabular}{lccc}
\hline \multicolumn{1}{c}{ Items } & $\begin{array}{c}\text { Controls } \\
(n=180)\end{array}$ & $\begin{array}{c}\text { TAD patients } \\
(n=206)\end{array}$ & $P$ value \\
\hline Age & $50.7(16-78)$ & $50.6(21-75)$ & NS \\
Sex (male) $n(\%)$ & $91(50.6)$ & $158(76.7)$ & $<0.0001$ \\
Smoking $n(\%)$ & $105(58.3)$ & $108(52.4)$ & NS \\
Diabetes $n(\%)$ & $27(15)$ & $20(9.7)$ & NS \\
Hypertension $n(\%)$ & $152(84.4)$ & $164(79.6)$ & NS \\
Dyslipidaemia $n(\%)$ & $101(56.1)$ & $110(53.4)$ & NS \\
CAD $n$ (\%) & $16(8.8)$ & $22(10.7)$ & NS \\
\hline
\end{tabular}

statistical differences between female patients and controls according to the dominant and additive model for rs2274756 (shown in Table 4). Finally, to account for the interference of potential confounding factors with our assessment of the relationships between the SNPs and TAD, we performed a multivariate logistic regression analysis that was adjusted for the covariates age, hypertension, diabetes, dyslipidemia, and smoking habit. We verified that the association between the rs2274756 gene polymorphism and TAD remained significant [odds ratio (OR) $0.30,95 \%$ confidence interval (CI) 0.11 to 0.79 ,

Table 3. Genotype distribution and allele frequency of all investigated single-nucleotide polymorphisms (SNPS) in thoracic aortic dissection (TAD) patients and control subjects. ${ }^{\# A c c o r d i n g ~ t o ~ t h e ~ a l l e l i c ~ t e s t ; ~}{ }^{\dagger}$ according to the additive model; MAF, minor allelic frequency (based on the whole sample).

\begin{tabular}{|c|c|c|c|c|c|c|c|c|c|c|}
\hline \multirow{2}{*}{ dbSNP ID } & \multicolumn{3}{|c|}{ Genotype (\%) } & \multirow{2}{*}{ MAF } & \multicolumn{3}{|c|}{ Genotype (\%) } & \multirow{2}{*}{ MAF } & \multirow{2}{*}{$P^{\#}$} & \multirow{2}{*}{$P^{\uparrow}$} \\
\hline & Patient & & & & Controls & & & & & \\
\hline rs3918249 & $\mathrm{TT}(17)$ & TC $(90)$ & CC (99) & $\mathrm{T}=0.301$ & TT (14) & TC (68) & CC (98) & $\mathrm{T}=0.267$ & 0.292 & 0.291 \\
\hline rs2274756 & $\mathrm{AA}(2)$ & $A G(46)$ & GG (158) & $A=0.121$ & $\mathrm{AA}(3)$ & $A G(52)$ & GG (125) & $A=0.161$ & 0.112 & 0.102 \\
\hline rs9609643 & $\mathrm{AA}(5)$ & $A G(57)$ & GG (144) & $A=0.163$ & $\mathrm{AA}(5)$ & AG (46) & GG (129) & $A=0.156$ & 0.789 & 0.789 \\
\hline rs8136803 & $\mathrm{TT}(1)$ & $\mathrm{TG}(16)$ & GG (189) & $\mathrm{T}=0.044$ & $\mathrm{TT}(0)$ & TG (19) & GG (161) & $\mathrm{T}=0.053$ & 0.555 & 0.557 \\
\hline
\end{tabular}

Table 4. Differences in the distributions of the rs2274756 genotypes and allele frequencies in the gender-stratified subsamples. "According to the allelic test; ' ${ }^{\top}$ according to the genetic models; OR, estimated odds ratio (for minor allele); DOM, dominant model; ADD, additive model; REC, recessive model.

\begin{tabular}{|c|c|c|c|c|c|c|c|}
\hline Group & \multicolumn{2}{|c|}{ Allele Frequencies } & $P^{\#}$ & OR (95\%) & \multicolumn{3}{|c|}{$P^{\dagger}$} \\
\hline TAD cases & 86.1 & 13.9 & 0.779 & $0.93(0.55-1.56)$ & 0.769 & 0.772 & 1 \\
\hline Controls & 85.2 & 14.8 & & & & & \\
\hline Controls & 82.6 & 17.4 & & & & & \\
\hline
\end{tabular}


$P=0.015]$ for female patients, but not for males.

\section{Discussion}

MMP-9 is a zinc-dependent proteolytic enzyme that is involved in degradation and remodeling of the extracellular matrix (ECM), which are essential steps in aortic dissection progression. Chen et al found that the MMP-9 -8202A/G polymorphism was associated with TAA and TAD in a white population ${ }^{[18]}$. In the present study, we found a significant association of SNP rs2274756 in MMP-9 with TAD in the female Chinese Han population, and this association remained significant independent of cardiovascular risk factors.

SNP 2274756 is located in exon 12 of MMP-9, where the mutation leads to a glutamine-to-arginine substitution at the 668th amino acid (Q668R) of the MMP-9 protein. This nonsynonymous substitution is situated in the coding sequence of the hemopexin domain ${ }^{[19]}$. Although our investigation is only a genetic association study and the precise impact of this polymorphism on protein function has not been confirmed by molecular biology techniques, several studies have focused on the computational prediction of the functional significance of this SNP with bioinformatic tools. According to ESEfinder, rs2274756 may change the binding motif, which could influence exon 2 splicing ${ }^{[20]}$, and Cotignola et al derived a synthetic structure of MMP-9 by 3D superposition of the different structural fragments of MMP-9 ${ }^{[21]}$. They suggested that an amino acid change of rs2274756 might alter MMP-9 function and/or interaction with its inhibitors. Nan et al computed SNP functional scores using several popular bioinformatics tools, including SNPs3D and PMut ${ }^{[22]}$. The results based on SNPs3D showed that the rs2274756 SNP was deleterious to the stability of the protein structure, and the MMP9 Arg668Gln is predicted by PMut to be pathological to protein function and by SNPs3D to be deleterious to the protein structural stability. This group demonstrated that rs2274756 was associated with the activity of the MMP-9 protein based on PMut and SNPs3D analysis. All of these findings could help explain the association between the MMP-9 SNP rs2274756 and the pathogenesis of TAD in females.

Furthermore, rs2274756 may not be singly causal but may also associate with the disease in more complicated ways. Linkage disequilibrium (LD) between this SNP and other causative SNPs cannot be ruled out. For example, the MMP-9 SNP rs3918241 was found to be in strong LD with rs2274768 $\left(D^{\prime}=1.0, r^{2}=0.97\right)^{[13]}$, and the promoter SNP rs3918241 was located within the core sequence of a GATA motif (GTAAAGGAAG[T/A]TAATTATCTC) that could bind GATA factors, which are master regulatory transcription factors important for the perpetuation and differentiation of human Th2 cells. Furthermore, several studies have suggested important regulatory roles for the Th1/Th2 cytokine balance in matrix remodeling and the pathogenesis of atherosclerosis and aortic aneurysm ${ }^{[23]}$.

Consistent with previous observations ${ }^{[24,25]}$, more male than female TAD patients were included in this study. To minimize the influence of gender difference and to detect a possible interaction between gender and SNP genotype status, we calculated associations with TAD by gender-specific, case-control analysis. We verified that the rs2274756 gene polymorphism was an independent, genetic susceptibility for females, but not for males. The reasons for this gender difference are not clear, and several factors have been proposed as possible causes of this phenomenon. Hormones may be an important factor, and MMPs have been found to be targets under hormonal control in previous studies ${ }^{[26,27]}$. MMP gene expression could be regulated by hormones ${ }^{[28]}$, which are quite different between men and women. Additionally, TAD is a complex trait, and susceptibility to TAD is influenced by a complex genetic background and potentially by environmental exposures. Gender differences in environmental factors, such as life habits and exposure to important risk factors, may contribute to this phenomenon.

In addition to TAD, the shifted balance of MMPs and TIMPs also plays an important role in some other tissue remodelingrelated diseases, such as primary angle closure glaucoma (PACG) and cancer. Awadalla et al reported an association between MMP-9 SNP rs3918249 and the pathogenesis of PACG $^{[12]}$, and Peterson et al demonstrated that SNPs rs9609643 and rs8136803 in the TIMP-3 gene contributed to individual differences in breast cancer susceptibility and survival ${ }^{[14]}$. However, we failed to find any significant differences in genotype or allele frequencies between the TAD cases and controls in our study. There are several possible explanations for this discrepancy. First, the associations between the tested SNPs that are involved in tissue remodeling-related diseases might be ethnic-specific. Awadalla et al conducted a study in a Caucasian population, but our study was performed in a Chinese Han population ${ }^{[12]}$. Second, the key mechanisms of TAD and other tissue-remodeling diseases are not exactly the same. For example, immunosuppression is crucial for cancer development, which is different from TAD formation ${ }^{[29]}$. Third, determining what causes these diseases is complex. Genetic variation and environmental factors may be involved in the pathogenesis of these diseases, and environmental risk factors between different populations could contribute to the discrepancy between the studies.

In conclusion, we investigated the potential association between four SNPs and TAD risk in a Chinese Han population. We demonstrated that the genetic variation rs 2274756 in MMP-9 gene was a susceptibility factor for TAD in the female Chinese Han subpopulation, and this genetic association was independent of the classical cardiovascular risk factors.

\section{Acknowledgements}

This study was supported by grants from the National Natural Science Foundation of China (81170283 and 81070251), the Beijing Natural Science Foundation (7112041), the Program for New Century Excellent Talents in University (NCET-11-0899), the Program for Changjiang Scholars and Innovative Research Team in University (IRT1074) and the Scientific Research Common Program of Beijing Municipal Commission of Education (SQKM201210025017). The authors thank all the volunteers 
who participated in the study.

\section{Author contribution}

Hong-jia ZHANG and Yi LV designed research; Xiao-long WANG and Ou LIU performed the experiments; Ou LIU, Yanwen QIN, and Hong-jia ZHANG analyzed the data; Xiao-long WANG, Ou LIU, and Hong-jia ZHANG wrote the paper.

\section{References}

1 Hagan PG, Nienaber CA, Isselbacher EM, Bruckman D, Karavite DJ, Russman PL, et al. The International Registry of Acute Aortic Dissection (IRAD): new insights into an old disease. JAMA 2000; 283: 897-903.

2 Tsai TT, Trimarchi S, Nienaber CA. Acute aortic dissection: perspectives from the international registry of acute aortic dissection (IRAD). Eur J Vasc Endovasc Surg 2009; 37: 149-59.

3 LeMaire SA, Russell L. Epidemiology of thoracic aortic dissection. Nat Rev Cardiol 2011; 8: 103-13.

4 Mizuguchi T, Collod-Beroud G, Akiyama T, Abifadel M, Harada $\mathrm{N}$, Morisaki $\mathrm{T}$, et al. Heterozygous TGFBR2 mutations in Marfan syndrome. Nat Genet 2004; 36: 855-60.

5 Bee KJ, Wilkes DC, Devereux RB, Basson CT, Hatcher CJ. TGFßRIlb mutations trigger aortic aneurysm pathogenesis by altering transforming growth factor $\beta 2$ signal transduction. Circ Cardiovasc Genet 2012; 5: 621-9.

6 Satoh K, Nigro P, Matoba T, O'Dell MR, Cui Z, Shi X, et al. Cyclophilin A enhances vascular oxidative stress and the development of angiotensin II-induced aortic aneurysms. Nat Med 2009; 15: 64956.

7 Liu O, Jia L, Liu X, Wang Y, Wang X, Qin Y, et al. Clopidogrel, a platelet $\mathrm{P} 2 \mathrm{Y} 12$ receptor inhibitor, reduces vascular inflammation and angiotensin II induced-abdominal aortic aneurysm progression. PLoS One 2012; 7: e51707.

8 Palavalli LH, Prickett TD, Wunderlich JR, Wei X, Burrell AS, Porter-Gill $\mathrm{P}$, et al. Analysis of the matrix metalloproteinase family reveals that MMP8 is often mutated in melanoma. Nat Genet 2009; 41: 518-20.

9 Kandalam V, Basu R, Moore L, Fan D, Wang X, Jaworski DM, et al. Lack of tissue inhibitor of metalloproteinases 2 leads to exacerbated left ventricular dysfunction and adverse extracellular matrix remodeling in response to biomechanical stress. Circulation 2011; 124 : 2094-105.

10 Liu CJ, Huang YL, Ju JP, Lu CL, Chiu AW. Altered transcripts expression of matrix metalloproteinases and their tissue inhibitors in tenon capsule of patients with glaucoma. J Glaucoma 2004; 13: 486-91.

11 Seiler R, Thalmann GN, Fleischmann A. MMP-2 and MMP-9 in lymphnode-positive bladder cancer. J Clin Pathol 2011; 64: 1078-82.

12 Awadalla MS, Burdon KP, Kuot A, Hewitt AW, Craig JE. Matrix metalloproteinase- 9 genetic variation and primary angle closure glaucoma in a Caucasian population. Mol Vis 2011; 17: 1420-4.

13 Beeghly-Fadiel A, Lu W, Shu XO, Long J, Cai Q, Xiang Y, et al. MMP9 polymorphisms and breast cancer risk: a report from the Shanghai Breast Cancer Genetics Study. Breast Cancer Res Treat 2011; 126: 507-13.

14 Peterson NB, Beeghly-Fadiel A, Gao YT, Long J, Cai Q, Shu XO, et al. Polymorphisms in tissue inhibitors of metalloproteinases- 2 and -3 and breast cancer susceptibility and survival. Int J Cancer 2009; 125: 844-50.

15 Liu O, Li JR, Gong M, Xu M, Du J, Zhang HJ. Genetic analysis of six SNPs in candidate genes associated with high cross-race risk of development of thoracic aortic aneurysms and dissections in Chinese Han population. Acta Pharmacol Sin 2010; 31: 1376-80.

16 Hiratzka LF, Bakris GL, Beckman JA, Bersin RM, Carr VF, Casey DE Jr, et al. 2010 ACCF/AHA/AATS/ACR/ASA/SCA/SCAI/SIR/STS/ SVM guidelines for the diagnosis and management of patients with Thoracic Aortic Disease: a report of the American college of cardiology foundation/American heart association task force on practice guidelines, American association for thoracic surgery, American college of radiology, American stroke association, society of cardiovascular anesthesiologists, society for cardiovascular angiography and interventions, society of interventional radiology, society of thoracic surgeons, and society for vascular medicine. Circulation 2010; 121: e266-369.

17 Nienaber CA, Fattori R, Mehta RH, Richartz BM, Evangelista A, Petzsch $\mathrm{M}$, et al. Gender-related differences in acute aortic dissection. Circulation 2004; 109: 3014-21.

18 Chen L, Wang X, Carter SA, Shen YH, Bartsch HR, Thompson RW, et al. A single nucleotide polymorphism in the matrix metalloproteinase 9 gene $(-8202 A / G)$ is associated with thoracic aortic aneurysms and thoracic aortic dissection. J Thorac Cardiovasc Surg 2006; 131: 1045-52.

19 Pinto LA, Depner M, Klopp N, Illig T, Vogelberg C, von Mutius E, et al. MMP-9 gene variants increase the risk for non-atopic asthma in children. Respir Res 2010; 11: 23.

20 Skibola CF, Bracci PM, Halperin E, Nieters A, Hubbard A, Paynter $\mathrm{RA}$, et al. Polymorphisms in the estrogen receptor 1 and vitamin $\mathrm{C}$ and matrix metalloproteinase gene families are associated with susceptibility to lymphoma. PLoS One 2008; 3: e2816.

21 Cotignola J, Reva B, Mitra N, Ishill N, Chuai S, Patel A, et al. Matrix Metalloproteinase-9 (MMP-9) polymorphisms in patients with cutaneous malignant melanoma. BMC Med Genet 2007; 8: 10.

$22 \mathrm{Nan} \mathrm{H}$, Niu T, Hunter DJ, Han J. Missense polymorphisms in matrix metalloproteinase genes and skin cancer risk. Cancer Epidemiol Biomarkers Prev 2008; 17: 3551-7.

23 Shimizu K, Mitchell RN, Libby P. Inflammation and cellular immune responses in abdominal aortic aneurysms. Arterioscler Thromb Vasc Biol 2006; 26: 987-94.

24 Giusti B, Saracini C, Bolli P, Magi A, Sestini I, Sticchi E, et al. Genetic analysis of 56 polymorphisms in 17 genes involved in methionine metabolism in patients with abdominal aortic aneurysm. J Med Genet 2008; 45: 721-30.

25 Bown MJ, Lloyd GM, Sandford RM, Thompson JR, London NJ, Samani $\mathrm{NJ}$, et al. The interleukin-10-1082 'A' allele and abdominal aortic aneurysms. J Vasc Surg 2007; 46: 687-93.

26 Wingrove CS, Garr E, Godsland IF, Stevenson JC. 17Beta-oestradiol enhances release of matrix metalloproteinase-2 from human vascular smooth muscle cells. Biochim Biophys Acta 1998; 1406: 169-74.

27 Grandas $\mathrm{OH}$, Mountain DH, Kirkpatrick SS, Cassada DC, Stevens $\mathrm{SL}$, Freeman MB, et al. Regulation of vascular smooth muscle cell expression and function of matrix metalloproteinases is mediated by estrogen and progesterone exposure. J Vasc Surg 2009; 49: 185-91.

28 Grandas $\mathrm{OH}$, Mountain DJ, Kirkpatrick SS, Rudrapatna VS, Cassada DC, Stevens SL, et al. Effect of hormones on matrix metalloproteinases gene regulation in human aortic smooth muscle cells. J Surg Res 2008; 148: 94-9.

29 Shiels MS, Engels EA. Increased risk of histologically defined cancer subtypes in human immunodeficiency virus-infected individuals: clues for possible immunosuppression-related or infectious etiology. Cancer 2012; 118: 4869-76. 